\title{
0 papel da atenção farmacêutica frente às interações fármaco-nutriente
}

\author{
The role of pharmaceutical care avoiding \\ drug-nutrient interactions
}

Recebido em: 29/07/2014 Aceito em: $06 / 11 / 2014$
Aline de Oliveira ANTUNES;

Ana Cristina LO PRETE

Universidade Guarulhos, UNG. Praça Teresa Cristina, 229, Centro, CEP 07023070, Guarulhos, SP, Brasil. E-mail: analoprete@yahoo.com.br

\section{ABSTRACT}

The pharmacist, through pharmaceutical care, may follow the pharmacotherapy, guiding and intervening when necessary, seeking improvements in health education and quality of life of the patient. The aim of this study was to review literature on the importance of pharmaceutical care to identify and solve drug-nutrient interactions, highlighting the implications of these interactions in the therapeutic effect of the drug or nutritional status. The research was conducted in books and scientific papers in databases such as Science Direct, Pubmed and Scielo between 2000 and 2014. The results showed numerous possibilities for drug-nutrient interactions, which can interfere with the effect of the drug and/or the nutritional state of the patient. Such interactions are often not identified, because the lack of knowledge of health professionals about the theme or due to the fasting requirement for bioavailability tests of drugs. Thus, the pharmacist, through pharmaceutical care and its extensive knowledge, may collaborate, reducing the risk of drug-nutrient interactions, increasing the patient's confidence and essence of the profession.

Keywords: pharmaceutical care; food-drug interaction; patient care

\section{RESUMO}

O farmacêutico, por meio da atenção farmacêutica, pode acompanhar a farmacoterapia, orientando e intervindo quando necessário, buscando melhoria na educação em saúde e na qualidade de vida do paciente. O objetivo deste trabalho foi realizar uma revisão bibliográfica sobre a importância da atenção farmacêutica na identificação e resolução das interações fármaco-nutriente, evidenciando as implicações dessas interações no efeito terapêutico do fármaco ou no estado nutricional. Foram realizadas pesquisas em livros e artigos científicos em bases de dados como Science Direct, Pubmed e Scielo, no período de 2000 a 2014. Os resultados mostraram que existem inúmeras possibilidades de interações fármaco-nutriente, as quais podem causar prejuízos ao efeito do fármaco e/ou ao estado nutricional do paciente, e essas muitas vezes não são identificadas, por falta de conhecimento dos profissionais da saúde ou devido aos testes de biodisponibilidade dos medicamentos serem realizados em jejum, por exigência dos órgãos sanitários. Desse modo, vê-se a necessidade da atuação do farmacêutico, que por meio do seu amplo conhecimento, pode colaborar com a diminuição dos riscos dessas interações fármaco-nutriente e nutriente-fármaco, resgatando assim a confiança do paciente e a essência da profissão.

Palavras Chave: atenção farmacêutica; interações alimento-fármaco; assistência ao paciente. 


\section{INTRODUÇÃO}

A atenção farmacêutica foi definida na Declaração de Tóquio em 1993 como a prática do profissional farmacêutico, no qual o paciente é o principal beneficiário. Essas ações englobam as atitudes, comportamentos, compromissos, inquietudes, valores éticos, funções, conhecimentos, responsabilidades e destrezas do farmacêutico por meio da farmacoterapia, com o objetivo de alcançar resultados terapêuticos uniformes e seguros na saúde e na qualidade de vida do paciente. A prática da atenção farmacêutica acontece por meio da orientação para educação em saúde, orientação farmacêutica, dispensação de medicamentos, atendimento, acompanhamento farmacêutico, registro sistemático de atividades e avaliação dos resultados, visando terapias eficientes e seguras $(1,2)$.

A atenção farmacêutica se encontra em constante fase de desenvolvimento e implantação no Brasil. A perspectiva do farmacêutico em sua atuação na atenção farmacêutica seria a de assumir sua responsabilidade e responder por seu compromisso, ao identificar e resolver todas as necessidades dos pacientes em relação à sua farmacoterapia (3, 4). Muitas situações críticas, tais como a insegurança e/ou falta de conhecimento em farmacologia dos prescritores, interferem negativamente no tratamento dos pacientes, que ficam expostos a possíveis interações fármaco-nutriente e nutriente-fármaco (5).

Neste contexto, as alterações dos princípios sobre os medicamentos, que não devem ser considerados apenas como insumos e sim um importante instrumento a ser utilizado no contexto multiprofissional, têm colaborado para mudar este panorama (6).

Um referencial dessa mudança é a Politica Nacional de Medicamentos (PNM), que tem como princípio garantir a segurança, eficácia e qualidade dos medicamentos, a promoção do uso racional e o acesso da população àqueles considerados essenciais (7). A PNM é muito complexa e, se colocada verdadeiramente em prática, poderá trazer melhorias significativas na qualidade de vida da população. As prioridades desta política são a revisão permanente da relação nacional de medicamentos essenciais (RENAME), a assistência farmacêutica, a promoção do uso racional de medicamentos e a organização das atividades de vigilância sanitária de medicamentos (8).

Neste âmbito o farmacêutico é o profissional apto a realizar e consolidar os princípios da PNM. Porém ainda é necessário que este profissional busque melhorias de seus direitos e realize com maior dedicação seus deveres na luta pelo uso seguro e racional dos medicamentos (8), que inclui a intervenção do profissional farmacêutico por meio da atenção farmacêutica, para o combate aos riscos não só entre medicamentos, mas também de interações fármaco-nutriente, visto que cada tipo de paciente utiliza um grupo diferente de medicamentos e uma dieta variada, existindo assim muitas possibilidades de interações, cada uma com seu risco específico.

\section{MÉTODO:}

Os dados coletados para o presente estudo foram obtidos em livros e bases de dados eletrônicas como Science Direct, Pubmed e Scielo, publicados entre 2000 e 2014. Para tal pesquisa foram utilizados, de modo integrado, descritores como atenção farmacêutica, interações alimento-droga e assistência ao paciente.

\section{RESULTADOS E DISCUSSÃO}

Interações fármaco-nutriente. A administração de medicamentos concomitante com alimentos é um fator capaz de determinar se a resposta terapêutica desejada ocorrerá adequadamente ou não. Desse modo, as prescrições realizadas devem ser preparadas com base em conhecimentos farmacocinéticos e farmacodinâmicos para evitar futuras interações com demais fármacos ou alimentos, sem interferir direta ou indiretamente no tratamento farmacológico (9).

A interação fármaco-nutriente pode ocorrer durante os processos farmacocinéticos e farmacodinâmicos, podendo gerar uma interferência do estado nutricional pelos fármacos e/ou interferência do fármaco pelo estado nutricional (9). Pode ocorrer por diversos mecanismos, como um desequilíbrio de nutrientes promovido por um medicamento, ou por meio da modificação de um efeito farmacológico pela ação e/ou presença de um nutriente (10).

Por meio da análise farmacocinética, pode ser constatado que as interações podem ocorrer em todas as fases (absorção, distribuição, biotransformação e excreção) (11).

Na primeira fase da farmacocinética, fatores como a competição pelo local de absorção, alterações de pH, diminuição da área absortiva de superfície pela presença de alimentos e alterações da motilidade intestinal podem alterar o processo de absorção dos fármacos, nutrientes ou ambos (12).

A via oral (v.o.) é a principal via de administração de medicamentos. Por este motivo se torna passível a essas interações fármaco-nutriente e nutriente-fármaco. No trato gastrointestinal ocorre a absorção do fármaco 
e do alimento, com possibilidade de existir competição e redução de absorção dos fármacos, nutrientes ou ambos $(11,13)$. A alteração do $\mathrm{pH}$ no trato gastrointestinal devido a alguns nutrientes pode influenciar na absorção de vários fármacos, pois muitos são bases ou ácidos fracos. A forma não ionizada será então mais rapidamente absorvida do que a ionizada, e essas alterações de $\mathrm{pH}$ podem gerar interações clinicamente importantes que devem ser previstas. Alguns exemplos da interferência da alteração do pH são as interações dos medicamentos AAS (ácido acetilsalicílico), ampicilina, isoniazida, penicilina $\mathrm{V}$ e rifampicina com leites e derivados, frutas e vegetais, alimentos que ocasionam a elevação do $\mathrm{pH}$ gástrico e redução da solubilidade dos fármacos, exigindo um intervalo mínimo de 2 horas entre a ingestão dos alimentos e a administração dos fármacos citados anteriormente. No mesmo contexto, alguns medicamentos podem alterar o $\mathrm{pH}$ estomacal, provocando a redução significativa da capacidade de absorção de nutrientes, como no caso da vitamina $\mathrm{B}_{12}$, que requer $\mathrm{pH}$ ácido para sofrer clivagem e ser absorvida $(12,14)$.

A presença de alimentos no estômago reduz o tempo do esvaziamento gástrico e a velocidade é limitada pela quantidade de quimo que o intestino delgado pode processar. Alguns exemplos são as interações entre AAS, amoxicilina, ampicilina, diclofenaco de sódio, digoxina, eritromicina, furosemida, ibuprofeno, levodopa, penicilina, tetraciclina com glicídios, lipídios e proteínas, nutrientes que geram o retardamento do esvaziamento gástrico, com consequente retardamento da desintegração e/ou dissolução dos fármacos, necessitando de um intervalo de 2 a 3 horas entre a ingestão alimentar e a administração de medicamentos $(14,15)$.

A alteração da motilidade intestinal pode melhorar a dissolução do fármaco aumentando o contato com a superfície absortiva, e por extensão, e a velocidade de absorção. Em outros casos, pose ser observada a diminuição de biodisponibilidade, devido ao aumento da velocidade de trânsito intestinal, o que diminui o tempo de contato para que ocorra a absorção $(13,14)$.

A interação fármaco-nutriente também pode ocorrer pela formação de complexos insolúveis, diminuindo a disponibilidade do fármaco e do nutriente. $\mathrm{O}$ exemplo mais conhecido é a interação de tetraciclina com íons metálicos, como o cálcio, encontrados em alimentos derivados de leite. O paciente deve realizar um intervalo de no mínimo 1 hora entre as administrações (12).

$\mathrm{Na}$ distribuição de fármacos, os nutrientes podem provocar mudanças nesse parâmetro farmacocinético, por diversas maneiras, enquanto que os fármacos não influenciam na distribuição dos nutrientes $(12,15)$.
Uma das formas de interações do processo de distribuição ocorre pelo deslocamento do fármaco de sua ligação das proteínas plasmáticas, podendo existir uma competição quando houver número limitado de sítios de ligação proteica. Um exemplo é a redução nas concentrações de albumina, ocasionando alteração da disponibilidade e farmacológica dos medicamentos $(9,12)$.

$\mathrm{Na}$ fase de biotransformação, os nutrientes podem inibir ou estimular o metabolismo do fármaco alterando a expressão e a atividade de sistemas enzimáticos de metabolização de fármacos, como o complexo P450. Os nutrientes podem então promover indução enzimática, necessitando de aumento da dose do fármaco, ou inibição enzimática, necessitando de redução da dose ou aumento do intervalo das administrações. Um exemplo é a interação de barbitúricos com dietas hiperproteicas e hiperglicídicas, que geram redução da atividade do CP450 , aumentando o tempo de efeito depressor $(12,14)$.

As interações fármaco-nutriente ocorridas no processo de excreção podem ocasionar aumento ou redução da taxa de filtração glomerular, reabsorção tubular ou secreção tubular ativa, ou por alteração do pH urinário. Alguns exemplos são a interação de medicamentos ácidos fracos como AAS e barbitúricos com alimentos básicos como leite, vegetais e frutas, que geram alcalinização da urina, redução da reabsorção tubular e aumento da excreção $(9,14)$.

As interações farmacodinâmicas entre fármaco e alimento ocorrem por meio da interferência da atividade fisiológica dos fármacos ou dos componentes alimentares. Podem ocorrer por sinergismo, ao aumentar a resposta terapêutica desejada, como no caso da associação de medicamentos depressores do sistema nervoso central e álcool. Podem ser também antagonistas, ao reduzir ou anular o efeito do outro, como a associação de dieta alimentar rica em vitamina $\mathrm{K}$ (verduras verde escuro, kiwi, ameixa, figo e abacate) com anticoagulantes cumarínicos, como a varfarina, que podem reduzir o efeito deste fármaco $(9,12,16)$.

Nos casos de interferências do estado nutricional pelos fármacos, um exemplo é a colchicina que gera deficiência de cálcio ao causar irritação no trato gastrointestinal, aumento da velocidade de trânsito intestinal e síndrome de má absorção. Nos casos de interferência no fármaco pelo estado nutricional temos como exemplo a alimentação hiperproteica com a administração do propranolol, que causa um aumento no tempo de meia-vida plasmática e prolongamento do tempo de efeito terapêutico (14). No entanto, em decorrência dos estudos realizados sobre possíveis interações serem escassos, e os testes de biodisponibilidade dos medicamentos serem 
realizados em jejum por exigência dos órgãos sanitários, as interações fármaco-nutriente são de difícil conhecimento, rastreabilidade e detecção (17).

Por meio dessas possibilidades de interações, o farmacêutico deve analisar e acompanhar cada paciente, por meio do seguimento farmacoterapêutico durante a atenção farmacêutica, avaliando o risco de interações fármaco-nutriente, o que podem ocasionar melhores resultados e efetividade do tratamento farmacológico após a intervenção. Caso ocorram interações, este profissional deve atuar junto ao prescritor para possibilitar a redução de tais situações negativas ao tratamento e à saúde do paciente (18). Desse modo, a prática da atenção farmacêutica constitui uma resposta efetiva para detectar, prevenir e resolver fracassos da farmacoterapia também no que se refere às interações fármaco-nutriente (18).

No entanto, grande parte das interações entre fármacos e alimentos acaba não sendo evidenciada ou notificada, devido à falta de estudos na área, e também pela negligência e falta de conhecimento dos profissionais da saúde, principalmente em âmbito hospitalar. Além disso, a ausência de profissionais farmacêuticos na equipe multiprofissional também contribui para que tais interações muitas vezes não sejam notadas e sanadas de forma adequada.

Nos últimos anos, muitas pesquisas vêm enfocando a ocorrência das interações entre medicamentos e alimentos, alertando sobre a incidência e complicações resultantes. Porém, o tema necessita de mais pesquisas mais aprofundadas pelos profissionais da saúde.

As interações fármaco-nutriente nos pacientes idosos. É importante lembrar que os pacientes idosos são os mais estudados em relação às possíveis interações fármaco-nutriente. $\mathrm{O}$ que justifica este fato é que são pacientes que geralmente fazem uso de polifarmácia e ingerem os medicamentos junto com os alimentos para facilitar a aderência (não esquecimento), o que aumenta significativamente o risco de interações. Além disso, os idosos são propensos a alterações e deficiências nutricionais por distintos fatores como desnutrição e desidratação, hipo-albuminemia e, consequente aumento da fração livre do fármaco, aumento da gordura corporal e redução do tecido muscular, deficiência nutricional de proteínas e micronutrientes, que podem alterar o metabolismo do fármaco; e alterações da função renal, ocasionando risco de toxicidade. As alterações supracitadas podem modificar os efeitos de agentes sedativos, cardiovasculares, diuréticos e outras classes terapêuticas muito utilizadas por idosos $(19,20)$.
Em um trabalho realizado com 73 idosos residentes em uma instituição de longa permanência no Paraná, foi observado que os medicamentos mais utilizados por estes pacientes foram fármacos que atuam no sistema nervoso central e cardiovascular. Em 166 prescrições, foram observados 28 medicamentos envolvidos em interações entre fármacos e nutrientes (21). Os principais casos apresentados foram de medicamentos contendo diazepam, nortriptilina, imipramida, pentoxifilina, lorazepam, haloperidol, ranitidina e clomipramina, que interagiram com cafeína, diminuindo a absorção. Os autores relataram que os medicamentos clorpromazina, metildopa, metformina e omeprazol ocasionaram diminuição da absorção da vitamina $\mathrm{B}_{12}$. $\mathrm{O}$ estudo ressaltou que além do prejuízo na farmacoterapia, podem ocorrer prejuízos na absorção dos nutrientes, aumentando o risco de desnutrição dos pacientes idosos (21).

No estudo realizado em Santa Cruz do Sul, Rio Grande do Sul, com 76 idosos hipertensos, com o intuito de verificar as possíveis interações fármaco-nutriente, foi observado que os grupos de fármacos com algum tipo de interação com os micronutrientes foram os diuréticos tiazídicos com 57,9\% dos casos, os inibidores da enzima conversora de angiotensina (ECA) com 47,4\%, betabloqueadores com $34,2 \%$ e os anti-agregantes plaquetários,com 32,9\% (22).

É necessário que ocorra melhoria na assistência prestada ao paciente, com boa orientação sobre os riscos das interações e a importância do ajuste dos horários das refeições e administrações dos medicamentos, o que facilita o entendimento dos idosos, diminuindo o risco de ineficácia na farmacoterapia. Os farmacêuticos, por meio da prática da atenção farmacêutica, devem investir no desenvolvimento de métodos para a melhoria da realidade da saúde dos idosos brasileiros, orientando de forma simples e clara estes pacientes, e também seus cuidadores, realizando o acompanhamento até total sucesso na farmacoterapia. Desta forma, os idosos terão melhor aderência ao tratamento medicamentoso, menores custos em medicamentos e internações, e assim, melhor qualidade de vida.

As interações fármaco-nutriente no paciente hospitalizado. No âmbito hospitalar, independente da idade e patologias do paciente, o farmacêutico é o profissional responsável por orientar tanto os internados quanto os pacientes ambulatoriais, buscando melhores resultados na terapêutica, redução de custos e uso racional de medicamentos (23). O papel do farmacêutico hospitalar além de administrativo é também clínico. Por meio da prática hospitalar da atenção farmacêutica, além de todos os benefícios já citados, deve ocorrer a integração 
do farmacêutico à equipe hospitalar, contribuindo assim com seus conhecimentos técnico-científicos, específicos da profissão farmacêutica (23).

Em um estudo realizado no Hospital Regional Justino Luz do município de Picos, no estado do Piauí, com 60 prontuários de pacientes internados, com o intuito de constatar possíveis interações fármaco-nutriente, foram observados 82 medicamentos prescritos, dos quais 16 apresentavam prováveis interações, totalizando 60 interações de fármacos com a dieta do paciente. Entre as interações identificadas as de maior frequência foram aquelas relacionadas aos fármacos captopril (dezoito interações), ácido acetilsalicílico (dez interações) e espironolactona (oito interações). Entre as interações relatadas, 32 ocorreram entre fármacos cardiovasculares e alimentos ricos em cálcio, como leite e queijo; treze interações entre anti-inflamatórios e alimentos como maracujá e alface; onze interações entre diuréticos e alimentos como leite, carnes, arroz e cenoura; e quatro interações entre fármacos que atuam no trato digestório com alimentos como carne, feijão, frango, abóbora e verduras (24).

No mesmo estudo foi revelado o grande risco que os pacientes internados sofrem ao apresentarem interações fármaco-nutriente ou deficiências nutricionais. Estes fatores podem aumentar o período de internação e custo do tratamento do paciente, e assim dificultar sua recuperação da saúde. Portanto, os dados sugerem que o trabalho multidisciplinar dentro do hospital, voltado às possíveis interações fármaco-nutriente dos pacientes internados, pode contribuir de forma satisfatória com a melhoria da qualidade de vida do paciente (24).

Outro tipo de paciente que necessita da atuação do profissional farmacêutico é o paciente que realiza também tratamento nutricional por meio da nutrição enteral ou parenteral. De acordo com o Protocolo de Terapia Nutricional Enteral e Parenteral, "a terapia nutricional poderá ser indicada nas seguintes situações: pacientes impossibilitados de ingestão oral adequada para prover de dois terços a três quartos das necessidades diárias nutricionais, seja por patologias do trato gastrointestinal alto, por intubação oro-traqueal, por distúrbios neurológicos com comprometimento do nível de consciência ou dos movimentos mastigatórios. Recomenda-se a terapia nutricional enteral e parenteral nos casos em que o paciente vem com ingestão oral baixa, por anorexia de diversas etiologias" (25). Segundo a Resolução $n^{\circ} 292$ de 24 de Maio de 1996, o profissional farmacêutico é o único profissional responsável por avaliar a prescrição médica da nutrição parenteral quanto à quantidade, qualidade, compatibilidade, interações e estabilidade; proceder e preparar a formulação (26).
Este profissional deve participar da comissão de Suporte Nutricional em cada unidade hospitalar para promover discussões de casos clínicos e atividades didáticas e científicas; e também participar, desenvolver e elaborar pesquisas em suporte nutricional (26).

O estudo realizado em várias unidades clínicas de um hospital de terceiro nível na Argentina, por meio da integração do farmacêutico na equipe nutricional, composta por médicos especialistas em endocrinologia e nutrição, enfermeiros e dentistas, mostrou a existência de diversos tipos de interações, inclusive as interações entre fármacos com nutrição enteral (27). Os pacientes estudados eram todos adultos, que utilizavam nutrição enteral, sendo um total de catorze pacientes, dos quais dez eram homens e quatro mulheres. Foram prescritos 77 medicamentos, sendo 51 via oral, 23 por sonda e três por via intramuscular (i.m). Os autores mostraram que foram realizadas doze intervenções farmacêuticas em relação à administração de medicamentos por sonda em doze dos pacientes (27).

Dentre essas doze intervenções, três delas foram decorrentes de interações fármaco-nutrição enteral, como a fenitoína (fármaco com janela terapêutica estreita), sucralfato e algeldrato, com a nutrição enteral dos pacientes. No estudo, os farmacêuticos propuseram a modificação da nutrição enteral para 1 hora antes e/ou 2 horas depois da administração do fármaco Este estudo comprovou a importância da presença do farmacêutico junto às equipes de suporte nutricional, colaborando com a resolução dos problemas derivados da administração dos medicamentos por sonda, podendo assim selecionar a nutrição enteral mais adequada para cada paciente (27).

As interações fármaco-nutriente nos pacientes pediátricos. A atuação do profissional farmacêutico direcionado ao paciente pediátrico também representa um enorme desafio, visto que as diferenças farmacodinâmicas entre adultos e crianças ainda necessitam de maior aprofundamento nos estudos em relação a diversos fármacos. Além disso, os pacientes pediátricos são mais suscetíveis a apresentarem eventos adversos graves (28).

Estudos realizados em uma clínica pediátrica de um hospital público no estado do Piauí, no período de agosto de 2010 e junho de 2011, com 113 crianças internadas submetidas a tratamento farmacológico por qualquer via e recebendo alimentação, por via enteral (v.e) ou parenteral, evidenciaram que as interações de maior frequência envolviam os antimicrobianos. com 79 casos no total; dentre eles a ceftriaxona (41 casos), ampicilina (12 casos), gentamicina (8 casos), cefalotina (7 casos), oxacilina (4 casos), cefalexina (2 casos), 
amoxicilina ( 1 caso), benzilpenicilina ( 1 caso), ciprofloxacino ( 1 caso), eritromicina ( 1 caso) e cloranfenicol (1 caso). As recomendações para o manejo dessas interações entre alimentos e antimicrobianos foram aumentar a ingestão de pré- e pró-bióticos, presentes em alimentos como iogurtes e produtos lácteos fermentados e nos componentes das fibras alimentares. Outros 22 casos de interações fármaco-nutriente ocorreram com corticoides, como a dexametasona (10 casos), hidrocortisona ( 8 casos) e prednisona ( 4 casos), que interagiram com alimentos ricos em vitamina $\mathrm{A}$ (retinol), $\mathrm{C}$ (ácido ascórbico), $\mathrm{B}_{6}$ (piridoxina), ácido fólico, $\mathrm{Ca}$ (cálcio), $\mathrm{K}$ (potássio), $\mathrm{P}$ (fósforo), $\mathrm{Mg}$ (magnésio), $\mathrm{Zn}$ (zinco), e tiamina, reduzindo a absorção das vitaminas $\mathrm{A}, \mathrm{C}, \mathrm{B}_{6}$, ácido fólico, $\mathrm{Ca}, \mathrm{K}, \mathrm{P}, \mathrm{Mg}$, e aumentando a excreção de vitaminas $\mathrm{B}_{6}, \mathrm{C}$, potássio, $\mathrm{Zn}$ e tiamina.

As recomendações para o controle das interações entre alimentos e corticoides foram aumentar ao consumo de frutas nos intervalos das refeições e evitar o consumo de laticínios após as refeições que contenham ferro. Os medicamentos anti-ulcerosos apresentaram nove casos de interações, entre eles o omeprazol (4 casos), ranitidina ( 3 casos) e cimetidina ( 2 casos), que interagiram com alimentos ricos em vitamina $\mathrm{B}_{12}$, reduzindo a liberação de secreção gástrica e dificultando a absorção de vitaminas $B_{12}$. As recomendações para evitar essas interações entre alimentos e anti-ulcera são aumentar a ingestão de alimentos ricos em vitamina $\mathrm{B}_{12}$, como ovos, leite e derivados, frutos do mar e fígado.

Um último e único caso foi de interação de óleo mineral com alimentos ricos em vitaminas A, D, E e K, que causou redução da absorção dessas vitaminas. A recomendação para este tipo de interação entre alimentos e óleo mineral seria de não ingerir alimentos ricos em vitaminas A, D, E e K junto ou próximo à administração da substância.Os autores expõem que todas as interações podem ser evitadas ou ao menos ter seus efeitos minimizados. Portanto, cada intervenção deve ser adotada individualmente em cada caso específico, reduzindo assim os riscos, custos e os períodos de internação dos pacientes (29).
O impacto das interações fármaco-nutriente na saúde do paciente e na prática da atenção à saúde ainda é desconhecida, principalmente aos pacientes idosos. No entanto, a análise desta questão através da prática multiprofissional com o acompanhamento farmacoterapêutico e nutricional é essencial para o sucesso da farmacoterapia (20).

De acordo com o exposto anteriormente, são inúmeras as atuações do farmacêutico, e também as possibilidades de interações fármaco-nutriente, as quais podem causar prejuízos ao efeito do fármaco e/ou ao estado nutricional do paciente. Desse modo, vê-se a necessidade da atuação do profissional farmacêutico, que por meio do seu amplo conhecimento, pode colaborar com a diminuição dos riscos dessas interações, ao utilizar uma de suas principais ferramentas: a atenção farmacêutica.

O acompanhamento farmacoterapêutico e nutricional tem impacto positivo no sucesso do tratamento farmacológico do paciente, ao identificar e resolver as possíveis interações indesejadas.

\section{CONCLUSÃO}

As pesquisas sobre interações fármaco-nutriente são ainda escassas em relação à grande incidência das ocorrências, talvez por despreparo de profissionais da saúde em identificar essas interações ou por falta de relatos devido ao fato de os testes de biodisponibilidade dos medicamentos serem realizados em jejum por exigência dos órgãos sanitários, dificultando seu reconhecimento.

O farmacêutico, com a atenção farmacêutica, é o profissional apto a oferecer o serviço de atenção voltada verdadeiramente ao paciente e seu tratamento farmacológico, e a resolver todos os problemas envolvidos neste ciclo e suas possíveis interações fármaco-nutriente e nutriente-fármaco.

Por meio da atenção farmacêutica, os farmacêuticos podem resgatar a confiança dos pacientes e principalmente, a antiga essência da profissão.

\section{REFERÊNCIAS}

1. OMS. El papel Del farmacêutico em la atención a la salud: Informe de La Reunión de la OMS. Tokio, Japão: Organização Mundial da Saúde. 1993.

2. Meneses ALL, Sá MLB. Atenção farmacêutica ao idoso: fundamentos e propostas. Geriatr. gerontol. 2010. 4(3): 154-161.
3. Oliveira AB, Oyakawa CN, Miguel MD, Zanin SMW, Montrucchio DP. Obstáculos da atenção farmacêutica no Brasil. Rev. Bras. Cienc. Farm. 2005. 41(4): 409 -413.

4. Cipolle RJ, Strand L, Morley PC. Pharmaceutical care practice: the clinican's guide. 2nd ed. New York: McGraw Hill, 2004. 
5. Consendey M, Veroneze C, Oliveira P, Espindola R. Avaliação do Conhecimento Sobre Interações Medicamentosas entre os Médicos Residentes de um Hospital Universitário do Sistema Único de Saúde Brasileiro. In: Concurso Público do I Prêmio de Incentivo à Promoção do Uso Racional de Medicamentos. 2009. Brasília: Ministério da Saúde; 2009.

6. OPAS. Atenção farmacêutica no Brasil: trilhando caminhos: relatório 2001-2002. Brasília: Organização Pan-Americana da Saúde. 2002.

7. BRASIL. Ministério da Saúde. Portaria No 3.916 , de 30 de Outubro de 1998. Dispõe sobre a Política Nacional de Medicamentos. Diário Oficial da União, $\mathrm{n}^{\circ} 215,10$ de novembro de 1998. Seção 1. p. 18 -22.

8. Margonato FB. As Atribuições do farmacêutico na Política Nacional de Medicamentos. Infarma. 2006. 8(3/4): 28-31.

9. Gomes MJVM, Reis AMM. Ciências farmacêuticas: uma abordagem em farmácia hospitalar. São Paulo: Atheneu. 2001.

10. Reis NT. Nutrição Clínica: Interações. Rio de Janeiro: Rubio. 2004.

11. Moura MRL, Reyes FGR. Interação fármaco-nutriente: uma revisão. Rev. Nutr. 2002. 5(2): 223-238.

12. Gennaro AR. A ciência e a prática da farmácia. $20^{a}$ ed. Rio de Janeiro: Guanabara Koogan. 2004.

13. Winstanley PA, Orme MLE. The effects of food on drug bioavailability. Br. J. Clin. Pharmac. 1989. 8(1): 621-628.

14. Oga S, Basile AC. Medicamentos e suas interações. São Paulo: Atheneu. 1994.

15. Guyton AC. Tratado de fisiologia médica. $8^{\mathrm{a}}$ ed. Rio de Janeiro: Guanabara Koogan. 1992.

16. Klack K, Carvalho JF. Vitamina K: metabolismo, fontes e interação com o anticoagulante varfarina. Rev. Bras. Reumatol. 2006. 6(6): 398-406.

17. De Fariña LO, Poletto G. Interações entre antibióticos e nutrientes: uma revisão com enfoque na atenção à saúde. Visão Acadêmica. 2011. 11(1): 91-99.

18. Hernández DS, Castro MMS, Dáder MJF. Método Dáder: manual de seguimento farmacoterapêutico. Terceira Edição (versão em português europeu). Granada: Universidade de Granada; 2009.
19. Olesen C, Harbig P, Buus KM, Barat I, Damsgaard EM. Impact of pharmaceutical care on adherence, hospitalisations and mortality in elderly patients. Int J Clin Pharm. 2014. 36(1): 163-171.

20. Faria MQ, Franceschini SCC, Ribeiro AQ. Estado Nutricional e Uso de Medicamentos por Idosos. Lat Am J Pharm. 2010. 29(1). 127-131.

21. Peixoto JS, Salci MA, Radovanovic CAT, Salci TP, Torres MM, Carreira L. Riscos da interação droga-nutriente em idosos de instituição de longa permanência. Rev. Gaúcha Enferm. 2012. (33)3: 156-164.

22. Weigel JA, Lepper L. Resposta da interação entre alimento e medicamento em idosos hipertensos do Projeto Pet-Saúde, em Santa Cruz do Sul-RS. Cient Ciênc Biol Saúde. 2012. 4(3): 141-148.

23. CRF-SP. Farmácia Hospitalar. São Paulo: Conselho Regional de Farmácia do Estado de São Paulo. 2012.

24. Lopes EM, Carvalho RBN, Freitas RM. Análise das possíveis interações entre medicamentos e alimento/nutrientes em pacientes hospitalizados. Einstein. 2010. 8(3pt 1): 298-302.

25. Júnior SJAM, Santos OF, Gomes MRL, Cabral LAF, Pereira PL. Protocolos de Terapia Nutricional Enteral e Parenteral. Teresina: Comissão Multiprofissonal de Terapia Nutricional. 2012.

26. CFF. Resolução no 292, de 24 de Maio de 1996. Ratifica competência legal para o exercício da atividade de $\mathrm{Nu}$ trição Parenteral e Enteral, pelo Farmacêutico. Conselho Federal de Farmácia Diário Oficial da União, 21 junho 1996. Seção 1. p. 11.123.

27. Sánchez AIG, DeAlmagro CGM, Aranzana MC, Continente AC, Hernández MAC. Atención farmacêutica en pacientes con nutrición enteral. Farm Hosp. 2006. 30(1):44-48.

28. Silva LIMM, Chaves BMM, Vasconcelos ASOB, Ponciano AMS, Reis HPLC, Fonteles MMF. O cuidado farmacêutico em pediatria. Rev. Saúde Criança Adolesc. 2011. 3(1): 66-69.

29. Lopes EM, Oliveira EAR, Lima LHO, Formiga LMF, Freitas RM. Interações fármaco-alimento/nutriente potenciais em pacientes pediátricos hospitalizados. Ciênc Farm Básica Apl. 2013. 4(1): 131-135. 